\title{
ISA - AN INVERSE SURFACE-BASED APPROACH FOR CORTICAL FMRI DATA PROJECTION
}

\author{
Lucie Thiébaut Lonjaret, ${ }^{\star \dagger, \circ} \quad$ Christine Bakhous ${ }^{\star} \quad$ Timothé Boutelier ${ }^{\star}$ \\ Sylvain Takerkart ${ }^{\dagger} \quad$ Olivier Coulon ${ }^{\dagger, \circ}$ \\ * Olea Medical, La Ciotat, France \\ $\dagger$ Aix-Marseille Univ., CNRS, Institut de Neurosciences de la Timone, Marseille, France \\ - Aix-Marseille Univ., CNRS, LSIS, Marseille, France
}

\begin{abstract}
Surface-based approaches have proven particularly relevant and reliable to study cortical functional magnetic resonance imaging (fMRI) data. However projecting fMRI volumes onto the cortical surface remains a challenging problem. Very few methods have been proposed to solve it and most of them rely on a simple interpolation. We propose here an original surface-based method based on a model representing the relationship between cortical activity and fMRI images, and a resolution through an inverse problem. This approach shows interesting perspectives for fMRI data processing as it is highly robust to noise and offers a good accuracy in terms of activations localization.
\end{abstract}

Index Terms - fMRI, surface-based method, inverse problem, signal reconstruction

\section{INTRODUCTION}

FMRI datasets are commonly analyzed in their acquisition space with volume-based methods. However, to hurdle the poor contrast to noise ratio (CNR) of fMRI data, these techniques usually apply a smoothing in the voxels space with very little consideration to the underlying brain anatomy, thus mixing informations that should not be (e.g. signals coming from opposite sides of a sulcus). Surface-based methods overcome this issue by studying the cortical signals in a domain approximating their original space : the cortical surface, hence keeping track of more relevant neighborhoods. Among those approaches, two classes of techniques can be distinguished. The conceptually simplest ones rely on interpolation methods, such as the trilinear interpolation [1], to transpose the 3D data onto the cortical surface. The more sophisticated processes embed anatomically informed models. Those models are then fitted to the fMRI 3D data [2] or used to interpolate the $3 \mathrm{D}$ data onto the cortical surface $[3,4,5]$. Because the acquisition system is responsible for transposing the cortical blood oxygen level dependent
(BOLD) signal onto a cartesian voxel grid, we propose an Inverse Surface-based Approach (ISA) to reconstruct the cortical activity onto the cortical surface through solving the corresponding inverse problem. ISA requires several inputs : a cortical mesh of the white matter / grey matter interface registered to the functional volumes, and the functional volumes corrected for head motion and slice timing. In this paper, we detail the construction of the forward model, introduce the corresponding inverse problem and present the methods to solve it. Several experiments are then conducted on both simulated and real datasets, that demonstrate the robustness of ISA to noise and its accuracy in terms of localization.

\section{METHODS}

\subsection{From cortical activity to fMRI volume : for-} ward model

The first step towards recovering the original cortical signal from MR images consists in defining the forward model (i.e. modeling how the acquisition process led the original cortical signals to the $3 \mathrm{D}$ images). This model should include both brain physiology and MR physics considerations, such as the shape of the BOLD signal, the partial volume effect (PVE), and the geometrical and architectural features of the cortex. We therefore started from the model exposed by Operto et al. [5]. This model suggests that signals $I_{S}$ on the cortical surface (represented by a mesh of $N_{n}$ nodes) at node $m$ result from a weighted sum of the cortical activities $a$ at neighboring nodes $n$ (Eq. 1), thus modeling the local induced activities by geodesic (i.e. following the cortical surface) weights $\omega_{\text {geo }}$ (see Fig. 1.b). It also supposes that the signals in the volume $V$ (divided in $N_{v}$ voxels) result from a weighted sum of the local cortical signals on the surface (Eq. 1), thus modeling the PVE by weights $\omega_{\text {norm }}$ which depends on the normal distance of a voxel $v$ to the 

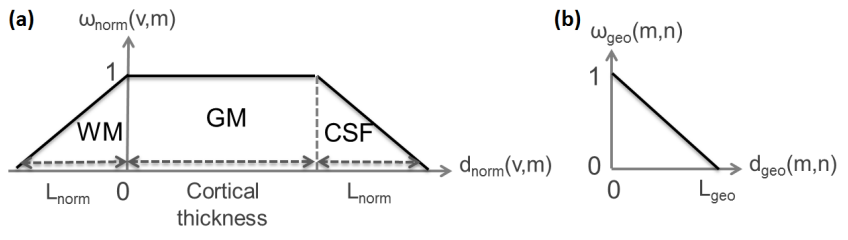

Fig. 1. Normal (a) and geodesic (b) components of the model designed by Operto et al. [5].

surface (see Fig. 1.a).

$$
\left\{\begin{array}{l}
I_{S}(m)=\sum_{n=1}^{N_{n}} \omega_{g e o}(m, n) a(n) \\
V(v)=\sum_{m=1}^{N_{n}} \omega_{\text {norm }}(v, m) I_{S}(m)
\end{array}\right.
$$

However this model suffers from some drawbacks. First, the cortical mesh often has a variable density, which results in each node representing portions of the cortical surface of various size. As the cortical mesh is a discretized version of a continuous surface, the cortical activity $a$ at a given node $n$ stands actually for all the cortical activities $\gamma(i)$ contained by the Voronoï cell $C_{n}$ associated to $n$. Because we have no prior on the distribution of those activities within $C_{n}$, we consider $\gamma_{\text {mean }}(n)$ the average activity over $C_{n}$ and obtain Eq. 2.

$$
a(n)=\operatorname{area}\left(C_{n}\right) \gamma_{\text {mean }}(n)
$$

Combining those considerations with Eq. 1 leads to Eq. 3 , thus taking into account the mesh variable density.

$$
\left\{\begin{array}{l}
V(v)=\sum_{n=1}^{N_{n}} M(v, n) \gamma_{\text {mean }}(n) \\
M(v, n)=\sum_{m=1}^{N_{n}} \omega_{\text {norm }}(v, m) \omega_{\text {geo }}(m, n) \operatorname{area}\left(C_{n}\right)
\end{array}\right.
$$

Second, we generalize Eq. 3 to time-series of volumes by simple concatenation (Eq. 4) to process simultaneously all the fMRI volumes of one session. Hence the forward model $M$ links real cortical signals $\Gamma$ and fMRI volumes $V$. Each column of $V$ represents the fMRI volumetric data at time $t$. Each column of $\Gamma$ similarly stands for the signals on the cortical mesh nodes $\gamma_{\text {mean }}$ at time $t$.

$$
V=M \Gamma
$$

\subsection{From volume to surface : inverse problem}

We solve the corresponding inverse problem, which consists in inverting the forward model (Eq. 4), with the method of regularized least-squares. The number of mesh nodes often exceeding by far the number of relevant functional voxels, our problem is highly underdetermined and thus ill-posed and ill-conditioned. Therefore we optimize a cost function composed of three terms, including two terms of regularization. The first term $f_{d}$ (Eq. 5) reflects how close the fMRI volumes predicted $\widetilde{V}$ from the forward model are to the observed data $V$. The noise covariance matrix $R$, estimated with [6], balances this term.

$$
f_{d}(\widetilde{\Gamma})=\frac{\operatorname{Tr}\left((V-M \widetilde{\Gamma})^{t} R^{-1}(V-M \widetilde{\Gamma})\right)}{2}
$$

To begin with, the cortical signal does diffuse locally [7]. This prior of smoothness can be modeled by a spatial regularization term $f_{s}$ (Eq. 6) relying on the geodesic distance between neighboring nodes. Here we introduce cliques notations. A clique $c_{i}$ is defined by a pair of cortical mesh nodes $\left(n_{i, 1}, n_{i, 2}\right)$ and a weight which depends on the geodesic distance $d_{g}$ between those two nodes and on a normalization term $D_{\text {norm }}$. Such a normalization term, based on the number $\Omega(n)$ of neighboring nodes of each of the two nodes of $c_{i}$, is necessary to cope with the mesh irregularity and ensure the same power of regularization at each mesh node.

$$
\left\{\begin{array}{l}
f_{s}(\widetilde{\Gamma})=\operatorname{Tr}\left(\widetilde{\Gamma}^{t} D^{t} D \widetilde{\Gamma}\right) \\
D_{i, j}=\frac{\delta_{j, n_{i, 1}}-\delta_{j, n_{i, 2}}}{d_{g}\left(n_{i, 1}, n_{i, 2}\right) D_{n o r m}\left(n_{i, 1}, n_{i, 2}\right)} \text { with } \delta_{j, l}=\left\{\begin{array}{l}
1 \text { if } j=l \\
0 \text { else }
\end{array}\right. \\
D_{\text {norm }}\left(n_{i, 1}, n_{i, 2}\right)=\sqrt{\frac{\Omega\left(n_{i, 1}\right) \Omega\left(n_{i, 2}\right)}{\Omega\left(n_{i, 1}\right)+\Omega\left(n_{i, 2}\right)}}
\end{array}\right.
$$

Furthermore, BOLD signals measured with fMRI come from the hemodynamic response function (HRF) which is smooth in time. Therefore, we define a temporal regularization term $f_{t}$ (Eq.7) with $T$ expressing the second order differential.

$$
f_{t}(\widetilde{\Gamma})=\operatorname{Tr}\left(\widetilde{\Gamma} T^{t} T \widetilde{\Gamma}^{t}\right)
$$

\subsection{Optimization}

The regularized least square estimator reaches the solution by minimizing the corresponding cost function where $\lambda_{D}$ and $\lambda_{T}$ are respectively the spatial and temporal regularization coefficients :

$$
f(\widetilde{\Gamma})=f_{d}(\widetilde{\Gamma})+\lambda_{D} f_{s}(\widetilde{\Gamma})+\lambda_{T} f_{t}(\widetilde{\Gamma})
$$

Minimizing this cost function amounts to nullifying its gradient, thus resulting in the following Sylvester equation (see [8] for more information on such equations) :

$$
H \widetilde{\Gamma}+\widetilde{\Gamma} G=K \text { with }\left\{\begin{array}{l}
H=M^{t} R^{-1} M+2 \lambda_{D} D^{t} D \\
G=2 \lambda_{T} T^{t} T \\
K=M^{t} R^{-1} V
\end{array}\right.
$$

Because operator $H$ is positive definite by construction and operator $G$ is semi-positive definite, we chose to use the linear conjugate gradients (CG) algorithm [9]. Indeed, this optimization technique is guaranteed to converge, under the previously mentioned conditions, to 
the unique solution, accurately and in a short amount of time.

Regularization coefficients were set as follows. In order to ensure the best spatial conditioning of the problem and guarantee an optimal convergence of the CG, $\lambda_{D}$ was chosen as to minimize the condition number (i.e. the ratio of the largest over the smallest eigenvalues) of operator $H$ (Eq. 9). Hence $\lambda_{D}$ becomes directly dependent of the mesh geometry and resolution.The optimal $\lambda_{T}$ was set empirically using an independent set of simulated data with meshes of various resolution. We found that the optimal $\lambda_{T}$ was quite stable across mesh resolutions and we set it at the empirical value of $\lambda_{T}=15$.

\section{RESULTS}

\subsection{Experiments}

ISA's performances were assessed on both simulated fMRI data, and real data. Simulated data were generated according to the following process. First, a cortical mesh was extracted from real data. Then we simulated a smooth activation blob of $1 \mathrm{~cm}$ of radius on this mesh, thus generating a surface map. Thereafter we constructed a block paradigm, using 10 repetitions of this map for the "off" blocks and multiplying these blocks by the desired percent of signal change (here PSC $=5$ ) plus 1 . Once the paradigm built, each node signal was convolved with SPM (http ://www.fil.ion.ucl.ac.uk/spm/) HRF model (considering a TR of $2 \mathrm{~s}$ ), thus producing a time-series of maps representing the BOLD fluctuations in time (Fig. 2.c - black). Finally, the forward model (Eq. 4) was applied to generate the corresponding fMRI volumes. Variable levels of Gaussian noise were added (Fig. 2.c - light grey) to get realistic fMRI data with variable CNR (i.e. the ratio of the highest temporal variations amplitude over the noise standard deviation). We conducted a noise robustness experiment on simulated fMRI volumes corresponding to 50 different realizations of activation blobs spread across the cortical surface for 3 levels of Gaussian noise $(\mathrm{CNR}=2.5,1.25$ and 0.25$)$. Finally, we produced a qualitative analysis of the spatial aspect of the projection using real data from a voice localizer experiment [10] : volumetric t-maps estimated using SPM, for the contrast 'voice - non voice', showing the individual location of the temporal voice areas, were projected on the cortical surface for four subjects. Results of all experiments were compared with those produced using the convolution kernels method that was shown to outperform all other methods [5]. These experiments used cortical meshes produced by the BrainVisa software (http ://brainvisa.info), with an average number of 60,000 nodes. Functional data had a spatial resolution of $3 \times 3 \times 3 \mathrm{~mm}^{3}$.
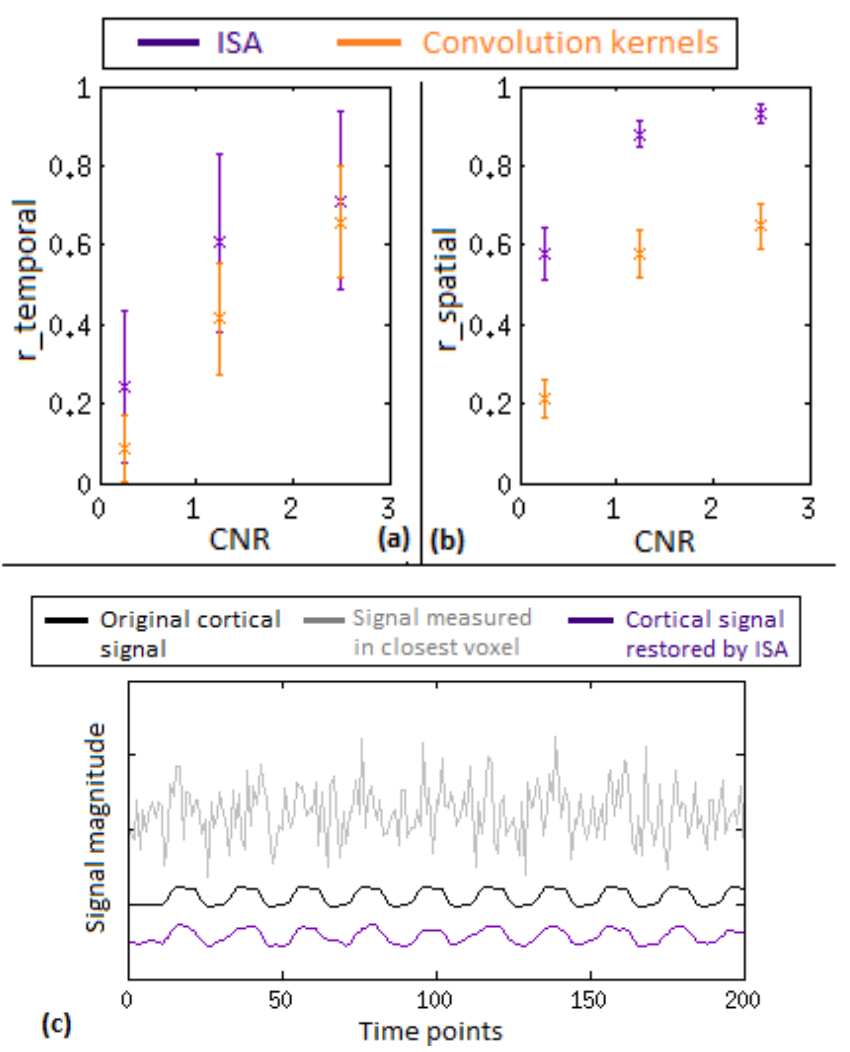

Fig. 2. Results of the robustness to noise assessment. Average and standard deviation of $r_{\text {temporal }}$ (a) and $r_{\text {spatial }}$ (b) over 50 realization. Qualitative representation of temporal activity at the center node of an activation blob (c).

\subsection{Robustness to noise}

Projection of time series was performed on all simulated datasets using ISA and the convolution kernels method. We evaluated the quality of the reconstructed signal in the spatial and temporal domains by computing the spatial Pearson correlation $\left(r_{\text {spatial }}\right)$ with the original spatial map before application of the forward model, and the temporal Pearson correlation $\left(r_{\text {temporal }}\right)$ with the original paradigm. For $r_{\text {temporal }}$ we considered only the temporal signals of the nodes localized within the activation area, whereas we considered the whole cortical surface for $r_{\text {spatial }}$. Under noisy conditions ISA outperforms the convolution kernels both in terms of localization and correlation to the experimental paradigm (Fig. 2.a,b). Indeed, we observe that ISA restores high values of $r_{\text {temporal }}$ even with poor CNR, and only starts to face real difficulties when the CNR goes below 1 , that is to say when the fMRI signal contains more noise than variations of interest. Furthermore $r_{\text {spatial }}$ is clearly higher for ISA than for the convolution kernels, showing a better control of the spatial regularization. It is important 


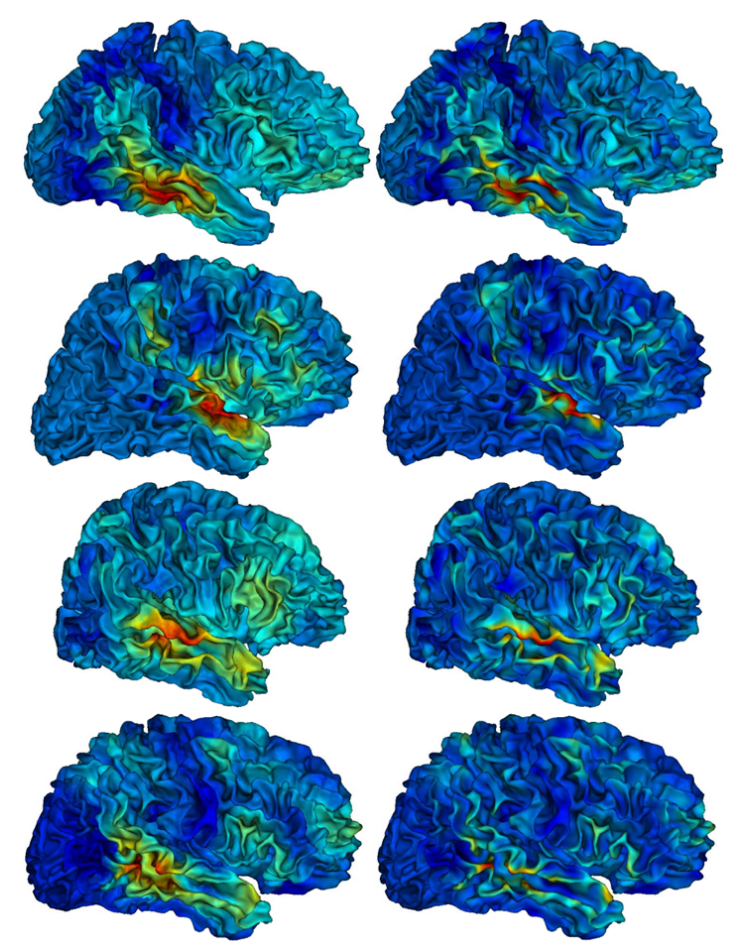

Fig. 3. Results of the convolution kernels (left) and ISA (right) applied on real data (colorscale is subject dependent).

to note that the spatial regularization term is set to optimize the spatial conditioning of the problem, but at the cost of a loss in signal magnitude restoration (Fig. 2.c).

\subsection{Real data}

Comparing the effect of ISA and the convolution kernels method, shown in Fig. 3, we noticed that there is an overall similarity of the activation patterns, but important local differences can be observed. In particular, it is clearly visible that peaks of activation are more focal with ISA. This shows a deconvolution effect that is intrinsic to the inverse problem approach : on top of ISA offering a more controlled spatial regularization, the forward model embeds several sources of signal mixing and the inverse resolution disentangle them. This could lead to a better spatial accuracy for functional data analysis.

\section{CONCLUSION AND FUTURE WORK}

We presented here a new surface-based approach to project fMRI data onto the cortical surface. This technique is highly flexible and offers a general framework where it is easy to introduce other prior knowledges as long as they can be expressed as a function. Moreover
ISA offers a good spatial and temporal accuracy even when faced to really noisy data. Future work will include experiments to assess ISA robustness to other kinds of errors arising from the preprocessing pipeline, such as anatomy-function registration errors or surface segmentation errors, and applications to real datasets.

\section{ACKNOWLEDGMENT}

We thank P. Belin for providing the 'temporal voice area' datasets.

\section{REFERENCES}

[1] A. Andrade, F. Kherif, J. Mangin, K. Worsley, A. Paradis, O. Simon, S. Dehaene, D. Le Bihan, and J. B. Poline, "Detection of fmri activation using cortical surface mapping," Human Brain Mapping, vol. 12(2), pp. 79-93, 2001.

[2] S. J. Kiebel, R. Goebel, and K. J. Friston, "Anatomically informed basis functions," Neuroimage, vol. 11, pp. 656-667, 2000.

[3] J. Warnking, M. Dojat, A. Guérin-Dugué, C. DelonMartin, S. Olympieff, N. Richard, A. Chéhikian, and C. Segebarth, "fmri retinotopic mapping-step by step," Neuroimage, vol. 17(4), pp. 1665-1683, 2002.

[4] C. Grova, S. Makni, G. Flandin, P. Ciuciu, J. Gotman, and J. B. Poline, "Anatomically informed interpolation of fmri data on the cortical surface," Neuroimage, vol. 31(4), pp. 1475-1486, 2006.

[5] G. Operto, R. Bulot, J. L. Anton, and O. Coulon, "Projection of fmri data onto the cortical surface using anatomically-informed convolution kernels," Neuroimage, vol. 39(1), pp. 127-135, 2008.

[6] John Immerkaer, "Fast noise variance estimation," Computer vision and image understanding, vol. 64, no. 2, pp. 300-302, 1996.

[7] P. B. Johnson, S. Ferraina, and R. Caminiti, "Cortical networks for visual reaching," Experimental Brain Research, vol. 97(2), pp. 361-365, 1993.

[8] J. D. Gardiner, A. J. Laub, J. J. Amato, and C. B. Moler, "Solution of the sylvester matrix equation axb $\mathrm{t}+\mathrm{cxd} \mathrm{t}=\mathrm{e}, "$ ACM Transactions on Mathematical Software (TOMS), vol. 18(2), pp. 223-231, 1992.

[9] S. Karimi, "Global conjugate gradient method for solving large general sylvester matrix equation," Journal of Mathematical Modeling, vol. 1, pp. 1527, 2013.

[10] P. Belin, R. J. Zatorre, P. Lafaille, P. Ahad, and B. Pike, "Voice-selective areas in human auditory cortex," Nature, vol. 403(6767), pp. 309-312, 2000. 